\title{
Structuring the Collaboration of Multiple Novice Design Ethnographers: Towards a New User Research Approach
}

\author{
Paul Gault ${ }^{1}$, Catriona Macaulay ${ }^{1}$, Graham Johnson ${ }^{2}$, and Judith Masthoff ${ }^{3}$ \\ ${ }^{1}$ School of Computing, \\ University of Dundee, \\ Dundee, Angus, DD1 4HN, UK \\ \{p.e.gault, c.macaulay\}@dundee.ac.uk \\ ${ }^{2}$ Advanced Technology and Research, NCR Labs, \\ NCR Corp, Dundee, DD2 4SW \\ graham.johnson@ncr.com \\ ${ }^{3}$ University of Aberdeen, \\ Meston Building, \\ King's College, Aberdeen, AB24 3UE \\ j.masthoff@abdn.ac.uk
}

\begin{abstract}
This paper proposes a new design research method to support businesses engaging in the innovation of products and services intended for use in public spaces. Increasing numbers of companies are turning to detailed user/consumer research often based on ideas from the design ethnography community. In an increasingly complex and fast moving business world, there is a need for faster user research that also provides a wider focus on the situation under investigation. A potential solution is using a larger number of fieldworkers on one study. As it would be difficult and costly to utilise many experienced design ethnographers, this may also involve the use of novices. This paper describes the development of a method for adapting existing practices to the emerging context outlined above (i.e. large numbers of fieldworkers, not all of whom necessarily have experience in ethnography). We discuss 3 field studies that show how the method can be applied and how it has been fine-tuned based on the outcomes. This method involves multiple groups of fieldworkers situated at a range of public spaces and each assigned with a specific theme of interest. The wealth of material that this fieldwork activity produces is then digested and insights are generated from it to help inform an understanding of existing behaviour within public space. This paper shows that fieldwork can be reduced to a set of simple tasks that can be successfully distributed over a group of novices facilitated by an experienced design ethnographer. This work will be extended further so that it can be applied as part of a toolkit for use in businesses where there is no established culture of utilising this type of user research.
\end{abstract}

Keywords: Ethnography, Crowdsourcing, Fieldwork, Innovation, Methodology, Public Space. 


\section{Introduction}

We will address the problem of providing ethnographic research for an increasingly complex and fast moving business world. This type of activity usually takes a long time to perform and is very specific in what it is observing. Our aim is to create a methodology to help explore the problem space in a short space of time with people who do not necessarily have a background in design ethnography. An objective of this is to have a broad focus on the subject matter so that there is a wide range of perspectives on what is being observed.

As a case study, we will try to identify opportunities for collaborative technologies situated within public space that encourage social interaction. Some examples of the kinds of new systems researchers and companies are interested in are digital signage, ubiquitous computing and mobile technologies. As public space is such a large context, we require a solution that will help us to understand the huge variety of activity going on there at any one time. Instead of a small number of fieldworkers taking many weeks to understand each of the perspectives of people who inhabit the space, a potential solution is to gain multiple viewpoints from many people making observations at the same time. We created a set of very broad themes that could be interpreted in multiple ways so that there would be a variety of approaches to gathering the fieldwork data. We will discuss the design and evaluation of the materials used to facilitate the fieldwork activity.

In essence, we will suggest a method for distributing fieldwork across a group of novice observers, reducing the fieldwork activity to a set of simple tasks. These tasks will be framed and facilitated by an experienced design ethnographer who will produce the support materials and lead the analysis workshops. The idea of using a group of people in fieldwork has similarities with Open Source development [1, 2]. In Open Source development, the community is encouraged to apply their skills to extend existing software. Our proposed methodology will provide more structure to the tasks requiring less expertise from participants. The idea of involving non-experts in fieldwork has similarities with crowdsourcing [3]. In crowdsourcing, well-defined tasks that are traditionally performed by employees are outsourced to an undefined large group of people via an open call. Our approach will not necessarily use an open call to recruit participants, and will involve more explicit continuous collaboration between the participants.

To illustrate our method, we will discuss a set of studies using observations of the interactions taking place in social spaces such as shopping centres, city squares and railway concourses. The outcomes of these studies provide an insight into how to design for such contexts along with techniques for managing this process.

In section 2 we will describe the other relevant literature that has helped to inform the development of our methodology. In section 3 we provide an overview for each of the main activities that constitute up our method. We explain how the method was first deployed in section 4 then subsequently refined and applied an additional two times in section 5. Finally, in section 6 we will detail the conclusions that were arrived at and indicate potential future work. 


\section{Related Work}

Design ethnography requires a set of custom techniques that can be adapted from their roots in anthropology to suit the requirements of new product development [4]. Previous studies have shown the application of ethnographic techniques using short timeframes [5] but the possibility of working with a number of fieldworker observations has not been explored.

We aim to use fieldwork to drive a broad exploration of the problem space. The analysis and synthesis of fieldwork observations will help to uncover new opportunities for the interactive systems we are interested in.

The normal limitations of fieldwork-based techniques include issues related to time, breadth and generalisability [6]. Fieldwork can be a very time consuming approach; doing both the observations and analysing the gathered data. It lacks breadth as it typically only studies one organization or culture in a specific time period, so offering in-depth knowledge only of particular contexts and situations. The generalisations arrived at from one study may be subjective as the researcher may have imposed an interpretation on what has been observed (different people may observe different things). We propose to use this subjectivity to our advantage as a way of channelling the observations from a variety of perspectives.

The initial stage of the methodology is the gathering of the fieldwork data. This will involve sending our participants out into a large public space with a set of themes that can help to guide the observations they make. Similar approaches [7] have shown that this technique will support the fieldworkers as they gather material by focussing on a specific topic of interest. Each theme will be explained using a reference card alongside a map of the physical space that they must make their observations within. The usage of reference cards to help explain a design methodology is a wellestablished concept [8]. We will use this technique as a way of co-ordinating the observations made by our group of novice fieldworkers.

For the second part of our methodology, we will undertake an affinity-mapping workshop where we analyze the fieldwork data using the initial steps of a grounded theory approach, namely open coding [9]. This is appropriate, as it will allow new themes to emerge from each fieldworker's individual field notes. The process of combining these themes will also allow us to arrive at a set of new-shared themes derived from all of the participants' fieldwork data.

Bringing together all of the field notes from the individual fieldworkers will involve the creation of an affinity-mapping diagram. Affinity mapping is originally based on the KJ-method [10], which develops themes into insights and helps identify the relationships among many issues. This technique will enable the group to consider the results of all of the observations made during the fieldwork activity. The outcome of this will be a KJ image that shows an externalized mental map of the fieldwork observations made by the group. This will help to show the broad themes relevant to the problem alongside the data they are derived from. This is a useful process as, by its nature, it is an activity that must be done fast encouraging a systemic response to the overall problem [11] instead of slowly going through with point-by-point solutions to each problem that is identified. During the workshops we will use the physical space to support the collaborative analysis work. The ability for large media to support many people collaborating in the same physical space [12] will help our 
participants arrive at a shared understanding of the fieldwork material they have collected. This will enable them to discuss the detail of each individual's fieldwork notes and relate this to the material that other participants have contributed.

In our method, we ask broad exploratory questions first. When working in an industry setting, it can be very difficult to be very specific at the outset. For example, "find the next killer application" might be the first question that a company asks when exploring a new area. By keeping it as broad as possible initially, this gives us enough space for any potential solutions (including unexpected ones) to be explored. The data analysis may result in more specific questions that can then be investigated in a new round of fieldwork.

\section{Proposed Methodology}

We will provide a brief summary of the method and then we will go into greater detail by explaining each of the main stages that were followed. Firstly, we broke down our problem of identifying opportunities for collaborative technologies situated within public space into 4 themes. We prepared materials for the participants to refer to whilst out in the field and assigned 2 person teams to work on each theme and placed them at different locations. The fieldwork observations took place on a single day where a large amount of data was recorded. This material was then brought back into the studio, shared with other group members and consolidated into a secure online space. Finally, a workshop was held where the group ruminated over the material they had gathered and some directions for further research exploration were identified. The four main phases of activity were:

- Materials preparation

- Fieldwork observations

- Dump \& distil

- Affinity mapping workshop

\subsection{Materials Preparation}

A set of briefing cards was designed for the participants to refer to whilst out in the field making their observations. This gave each of the teams a key area to focus on both conceptually and physically. One side of the card detailed a research theme with a title and a set of questions whilst the reverse of the card had a map marking out where the observations were to be located. The research theme was a title followed by single sentence along with 3 example questions loosely related to the theme. The cards were A5 size so they were small enough for the participants to keep in their pocket and so easily referred to if needed. The cards were also laminated to make them robust in case the weather conditions were not favourable.

\subsection{Fieldwork Observations}

This phase of work is where the participants go out into the field and make their observations. The participant group were split into equal numbers of people and each 
team was given a briefing card containing a specific theme. The whole group were briefed before setting off to make their observations. It was suggested that one person take the role of writing notes and another person of capturing visual material. If it was felt necessary, the team members were free to switch roles at any point during the exercise. The importance of keeping communication flowing between members was stressed so that there was a shared plan of action for what was being observed. The teams were encouraged to take plenty of breaks, as making the observations can be quite an intensive exercise. During these breaks, they were able to use the opportunity to talk about how the task was progressing. The teams were asked to keep any observations in context so that they were mindful of the environmental conditions, time of day and density of people in the space they were observing.

\subsection{Dump and Distil}

This is the process of putting all the fieldwork data into a form so that it can be worked with further. It began as soon as the observation activity was completed. The teams were asked to write-up another version of the scratch notes they had recorded immediately after making their observations so that nothing was forgotten, as this would easily happen otherwise. The whole group came together to discuss their observations and reflect upon the process they had gone through whilst out in the field. From the outset, the group were encouraged to remain analytical about their observations by not jumping ahead into thinking about designing new solutions. They were asked to discuss their strategy throughout the task, how they interpreted the individual research questions and how they approached working in each location. The group were guided to create backups of all their notes in case they were lost and also, they could be easily shared with each other. To facilitate this, secure network drives were created where everyone was able to pool their data for further analysis.

\subsection{Affinity Mapping Workshop}

The final part of our method involved a workshop to help reach a shared understanding of the material that had been gathered. The teams were asked to make a pass through their fieldwork data and extract items of interest onto post-it notes. Each team was guided to use 1 colour of post-its and initial each note so that if necessary, the data could be queried later on in the process. Once the groups had written out their notes, they started to arrange them into sub-groups and used index cards to write headings above the groupings. A photograph of each team's notes was taken at this point so if needed, the layout of notes was saved and would be referred back to later on. The final part of the workshop involved all of the teams pooling their data together to see what overall findings could be arrived at. This involved taking down the sub-headings from each group and arranging these into super-headings across all of the groups. The original post-it notes were pulled into these new super-headings so it could be seen how appropriate they were. It should be stressed that this activity can be repeated a number of times to see if the same or a different understanding is reached. 


\section{Field Study 1}

\subsection{Study Design}

Following the results of our previous Urban Traces study [13], we decided to develop our methodology by creating a number of themes and targeting specific physical spaces related to them.

4.1.1 Questions. The research questions for the fieldwork observations were split into 4 different themes. Each theme was explained using a sentence outlining the focus of the observations and then 3 follow-up questions that the participants were asked to try to answer. The themes, explanations and questions were as follows:

Connections. Observe how people navigate their way through physical public space using the way finding systems that are present. How useful are the existing way finding systems? Where do clusters of people and flows of activity occur? What would happen if the way finding system were to fail?

Transactions. Observe how the exchange of information, goods \& services is facilitated. What are the social norms and practices taking place around self-service kiosks? How are people using public space as a platform for collaboration? Where is technology being used and why?

Interactions. Observe how people interact both with each other and with the systems that are present in public space. How does the space affect the way in which people behave? Where is one-to-one \& one-to-many communication occurring? What kinds of technology are present and why are they there?

Conversations. Observe the patterns in behaviour for ways in which people congregate in public space. What are the differences between how large groups of people behave in comparison to small groups \& individuals? How does the physical layout affect places that people gather? Where are the empty spaces and why are they so?

4.1.2 Participants. Fourteen students on a Masters of Design Ethnography (MDE) participated in the study ( 4 male, 10 female). The course teaches students to perform user research and explore how this is incorporated into the work of companies globally. The participants were grouped into teams of 2 so that the different activities could be shared out within each team.

4.1.3 Location. The city centre of Glasgow was used to carry out the fieldwork activity. Glasgow is the largest city in Scotland so there would be a relatively high volume of activity on the day of the fieldwork. Four separate physical spaces were set out to match the research themes. The connections teams were located in Glasgow Central train station; the transactions teams were located in a shopping centre called The Buchanan Galleries; the interactions teams were located in Buchanan Street and the conversations team was located in George Square. Prior permission was sought to make observations in the train station and shopping centre locations. No permission was given to take photographs or record video in these locations, so these particular teams had to rely on note taking and sketches to record their observations. 
4.1.4 Materials. Each group was given a plastic wallet containing: a briefing card, sketchbook, notebook and pencil.

4.1.5 Procedure. The study was split into 3 sessions:

- Fieldwork: a trip to Glasgow to carry out fieldwork observations.

- Dump \& distil workshop: The group discussed the process of carrying out the observations and explored ways of presenting the gathered material.

- Affinity mapping workshop: Participants analyzed and synthesized the fieldwork notes and other types of data. The purpose was to arrive at a set of insights based on the observations that each team had made. Following on from this, the group tried to make overall sense of activities that had gone on that day across all of the original themes.

\subsection{Fieldwork}

The fieldwork activity began with a briefing in the city square. Participants were put into pairs, creating 7 teams and then the briefing packs were issued. The teams were given the option of assigning roles themselves but it was suggested that they split up the activity so that one person had the role of keeping notes and the other person the role of drawing sketches visually representing any observations. They were encouraged to keep communication flowing between each other whilst working on this activity.

The teams were asked to use the materials provided within the pack to help record their observations. The interactions and conversations teams, who were working outside, were also asked to use a digital camera to take photographs. The transactions and connections teams were working inside so had to rely solely on written notes and drawing sketches.

We scheduled 2 bursts of activity to take place on the day of the fieldwork. The first period lasted an hour and half, which allowed each of the teams to become acquainted with the research questions and familiarised with the locations where they were observing.

After the first burst of activity, the group gathered at a cafe to discuss how the fieldwork was going. This enabled them to talk about any problems that had occurred in the first pass of their separate spaces. Some issues that arose from this discussion were:

- Feeling overly visible whilst note taking and strategies for taking notes discretely.

- Having difficulty to decide what to focus on when there was too much happening at once to be able to record everything.

- Pretending to do something else other than observing so that the notes could be memorised and then recorded shortly after.

Once the group had finished their discussion, they split up once again into their individual teams and set off back to their designated spaces. This gave them another hour to make some further observations. 


\subsection{Dump and Distil Workshop}

A week after the fieldwork, participants were asked to discuss how they managed the fieldwork process and how they interpreted the research questions that were set. They also answered questions related to the design of the study, the location and their use of the materials provided.

4.3.1 Approaches to Fieldwork. There was a mixture of approaches to making the observations in pairs. The conversations team decided to stay together throughout the fieldwork activity. This gave them the opportunity to have a continual dialog about what was being observed. Conversely, both the interactions teams separated whilst taking field notes and regrouped at set time intervals to discuss strategy about where to focus observations next (Fig. 1). A common approach was to take an initial walk through the space to become familiarised with it and then plan how to break their observations down into manageable chunks of activity. One of the interactions teams did this by splitting the timing of their observations equally for separate parts of the street so that they could systematically move down the street in 20-minute intervals.

All of the participants seemed to follow the instructions to allocate specific roles to each team member. There was commonly someone within each team who was stronger at recording things visually in comparison to taking written notes. Many of the teams switched roles at various points to try and gain a different perspective on the activity although, in relation to their drawing abilities, this meant that some team members were slightly outside of their comfort zone.

Where there were 2 teams working within the same space, they negotiated amongst themselves to try and eliminate the possibility of a crossover. For example, the 2 teams working on the transactions theme inside the shopping centre deliberately went to the highest and lowest floors so that more ground could be covered in the time available.

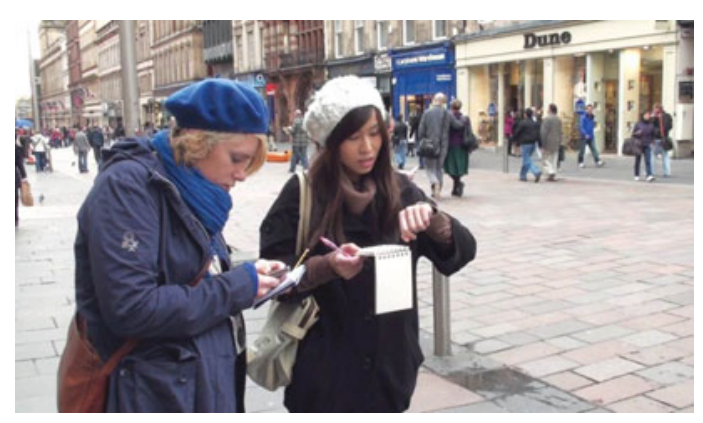

Fig. 1. One of the Interactions teams working out a strategy

A common insight was the groups finding it beneficial to have a higher point of view for making their observations from. The connections teams made use of the balconies that were available in the train station and the interactions teams used a set of steps outside a concert hall to gain a better vantage point. The teams felt that this provided a better overview of the activity that was occurring. 
Many of the participants noted that they would like to return to the spaces again to go over some of the details that they might have missed the first time around. For example, checking on the sequence of events that a person who is interacting with a self-service kiosk has gone through. They also thought that returning at various times of day when the spaces are quieter or busier would be useful.

4.3.2 How to Share Data. The next step was to discuss how to go about sharing the fieldwork data between all of the teams. One of the participants suggested using a large physical map of the location to annotate their observations with over time. This might have been difficult for some of the interior spaces where maps were not so easily available. This also jumped too quickly to a physical space metaphor for understanding the data whereas at this stage, we needed to remain thinking about the data in a more abstracted way.

A secure virtual sharing space was set up for participants to place their fieldwork notes and images into in preparation for the final analysis workshop. This gave the participants an opportunity to see how other teams had approached the problem of how to keep easily readable field notes and the recording of other fieldwork data. The total amount of fieldwork data uploaded to the sharing space was 55 sketches, 175 photographs and 33 pages of notes. Each of the participants used a login name to sign into the shared virtual space so that the ownership of the content could easily be tracked. The fieldwork data was uploaded in a variety of formats ranging from single image files to full PowerPoint presentations. A standard format should have been agreed beforehand to make it easier to sort through the data in preparation for the workshop. Also, it would have been much easier to process the images if the individual instances of the image files were uploaded instead of, for example, embedding them within a PDF.

\subsection{Affinity Mapping Workshop}

In order to make sense of the vast amount of gathered fieldwork data, a final workshop session was held. The aim was to quickly reduce all data into a set of shared insights across all themes from the previous fieldwork activity. Given the time constraints, affinity mapping was selected as a suitable method.

The affinity mapping involved extracting notes and ideas from a larger piece of text or data then writing them onto sticky post-it notes. Once this was done, the next step was to try and organize the notes into sub groups to see if new themes emerge based around the particular notes that have been extracted. The workshop plan was to repeat this grouping process multiple times. Firstly, working within each of the small teams of 2 participants. Then working with both teams across each theme and finally everyone working across the overall themes.

4.4.1 Workshop Materials. There were a number of materials that were necessary to effectively carry out the workshop: a supply of post-it notes in 4 different colours; white index cards for recording theme headings; 8 sheets of A1 black paper; marker pens and drawing pins. Also, the day before the workshop, the group was asked to print out and bring along a hard copy of their fieldwork notes. A selection of the visual material that was uploaded to the shared folder was printed out and pinned onto the walls. 
4.4.2 Field Data Tagging. Each of the teams was asked to make a pass through the opposite team's field notes working within the same theme. Each piece of extracted data was written onto a single post-it note and stuck onto a sheet of black paper in no particular order. The teams were asked to stick to one colour of post-its so where the note had come from it could be traced. The purpose of this exercise was to allow the team to offer a different perspective on someone else's field notes. This also meant that the field notes had to be written in a clear enough way so that other people could make use of the data.

4.4.3 Grouping Data. The participants returned to the post-it notes that had been recorded for them by the other team. They were then asked to read through the notes and start grouping these together as they deemed appropriate. Headings for each of the groups were written onto index cards and placed above them.

4.4.4 Grouping Themes. This task involved asking the 2 groups working on the same theme to bring together their notes to see where the commonalities were between them (Fig. 2). The two sheets of black paper were joined together so that there was a shared space for the groups to work within. This exercise allowed the teams to filter out duplicate sets of groupings. It also required the groups to explain the notes that they had written for each other previously and come to a shared understanding about the activity going on in each of the physical spaces they had observed.

The ways in which the groups manipulated the post-it notes in the space available was interesting. One of the groups moved all of their notes onto one side of the black sheets so that they had a clear space to organise them within. They could also be seen placing groups of notes at an angle on the paper as a way to differentiate them from the others. Once each of the teams had finished grouping their boards, they were photographed as a way of saving the layouts so that they could be referred to again later.

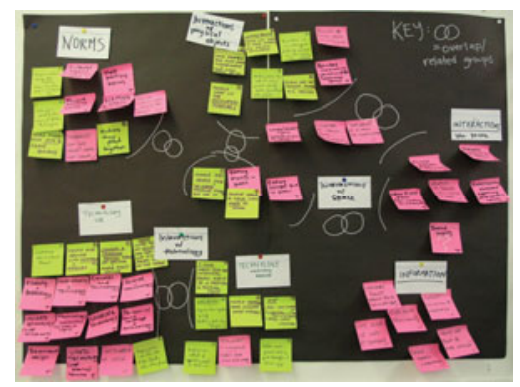

Fig. 2. The Transactions team's board showing their integrated data headings

4.4.5 Extracting Super Themes. At this stage, we had to zoom out of the original themes and try to arrive at an overall understanding of the activity that was taking place in the city centre on that particular day. The participants were given half an hour to look over the notes and themes that all the other teams had arrived at through the 
previous set of activities. All of the headings that were written onto the index cards were pulled off the wall and collected into a pile. The participants then gathered around a large table and all of the headings were laid out onto it. This allowed everyone to see where there might be some overall groupings that the headings could fit into. The headings that were duplicates and those that were quite vague, for example "Technology" were removed from the table.

Following a brief discussion amongst the group, it became apparent that there was a clear split between the interactions that people have with each other and their interactions with space and technology. The participants allocated separate areas of the table for each of these groupings and set about putting each of the headings into these spaces. The interactions that people have with each other grouping was given the title "Interpersonal Interactions" and 12 headings were placed under this. The interactions that people have with space and technology grouping was given the title "Ways of Interacting" and 26 headings were placed under this. A photograph of each of the groupings was also taken so that they could be referred to again later on.

4.4.6 Evaluating Super Themes. In order to find out if the themes that we had identified were appropriate, we had to refer back to our original post-it note field data. This would allow us to see how all of this data would fit into the new super themes that we had identified. The participants were instructed to split themselves into 2 large groups and divide the space in half down the middle of the table.

This provided the group with another new space to think about all of the gathered data that had emerged from an attempt to organise it under the super headings. The next stage of analysis for the participants would be to try to map all of the post-it notes along each of these newly identified axes. The workshop had already ran an hour over the original scheduled time at this point so was drawn to a close. Photographs of all the post-it layouts at the end of the session were taken to document how far the participants had progressed with analysing the data.

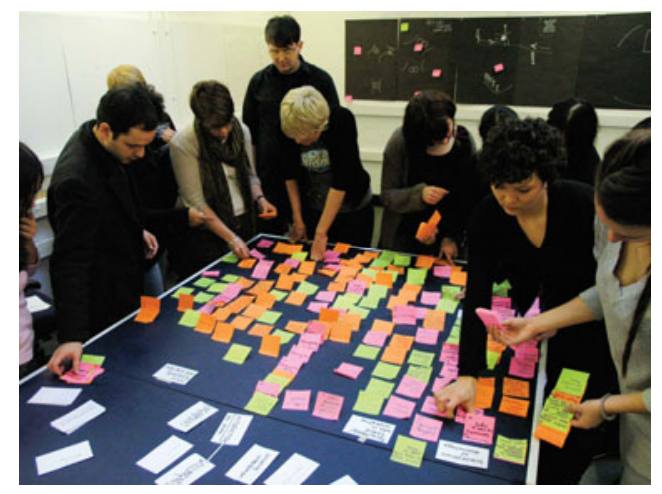

Fig. 3. Arranging the data into the super themes

The groups were then asked to pull all of the notes off the wall and place them under each of the super themes (Fig. 3). This enabled the groups to start from scratch again in relation to how they perceived the individual pieces of data. This introduced 
them to the idea of not being too precious about the original groupings they had set out and start thinking in new ways about organising the data.

This exercise enabled the group to see how valid each of the super headings were. The team working on the "Ways of Interacting" super theme split their groupings up again into 2 which were "Interactions with Technology" and "Interactions with Space". Also, some of the notes were split between the 2 super themes so were placed along a line down the middle between the spaces.

4.4.7 Identifying Axis. Due to the limitations of the tabletop space, the group quickly started to run out of room. In order to lay the data out more coherently, one of the participants suggested the need to arrange the data along different physical axis. Coincidentally, within the studio space there was a set of wooden exhibition stands. The participants used masking tape attached to them to mark out separate axis. These axes were 'person' to 'people' along the $\mathrm{X}$-axis, 'tech' to 'non-tech' along the Y-axis and 'macro space' to 'micro space' along the Z-axis (Fig. 4).

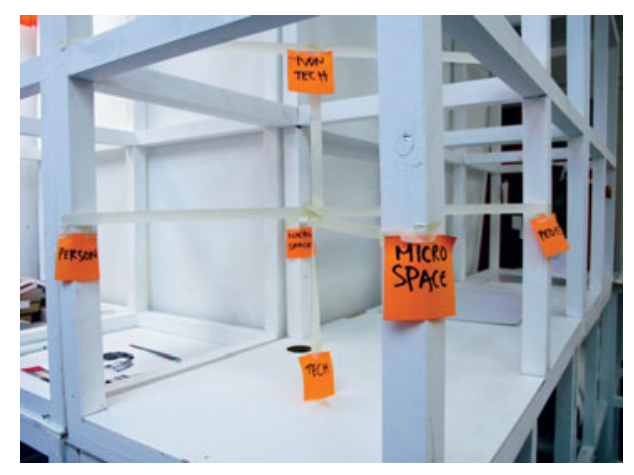

Fig. 4. The axes the workshop participants identified

\subsection{Navigating the Data}

Once the workshop had been completed, there was a vast amount of data that would help to map out the problem. These were: the raw fieldwork notes \& sketches; the digitised written up notes, photographs of the affinity boards at different stages of the workshop, the axis model, the unsorted post-it notes and index card themes.

When looking through this data, there seemed to be a regular need to zoom in and out of the different levels of material that was gathered. The different levels of information that are regularly accessed include:

- themes - top level providing colour-coded groupings of all themes created during the affinity mapping process.

- groupings - themes that have grown out of the arrangement of the individual tags.

- tags - recorded tags capturing any individual ideas based on an initial survey of the source material.

- source - the original field notes, sketches, images, sounds or video recorded whilst out in the field. 


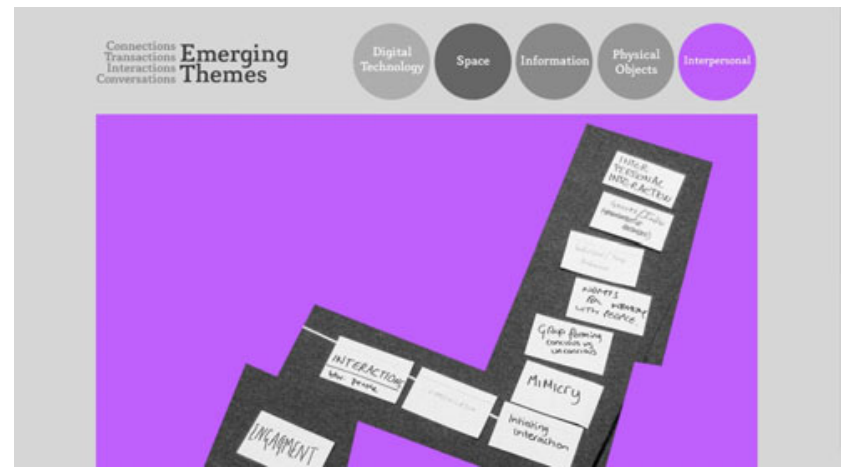

Fig. 5. A screenshot of the prototype tool

A web-based application was designed to prototype how these levels of information might be interacted with using a screen-based tool (see Fig. 5). The gathered material from the affinity-mapping workshop was used as example content to build this tool around. This prototype was only a sketch of an idea for how such a tool might operate. Future work requires enhancing the interface to allow zooming interaction for the content and adding the database backend to help manage the different types of media. In addition, user evaluation is needed.

\subsection{Reflecting on the Method}

The week after the affinity workshop, interviews with four of the participants were held to discuss their experience of working with the fieldwork data in a large group. The outcome of this discussion helped us to identify a number of points that could be explored further with the future iterations of the methodology. The issues we have identified were:

- Sharing context

- Descriptiveness of field data

- Physical access to the affinity boards

- Information overload

- Working across the different axis

4.6.1 Sharing Context. As the two teams worked together under each theme, there was a sense of familiarity with the field notes and being aware of where they had come from. An interviewee working on the connections theme noted that the opposite team had observed the same activity from another perspective so this helped to validate their own insights further. This had felt like they were "reading a guide book for a city they had visited before" so there was a clarity that could be gained from analyzing these observations.

It helped that the teams were working in the same place in the same time frame so they were able to crossover and relate perspectives. If they had to work with a group that had been located at different locations, it might have taken much longer to explain some of the meaning of what had been observed. 
4.6.2 Descriptiveness of Field Data. When reading through other team's field notes, there was a reoccurring need to have set out a standard way for writing clear field notes so that others can make use of it more easily. Some sets of notes were written like a story in the format of long paragraphs. There is a need for a shared format that requires the fieldworker to indicate timings next to each of their pieces of data along with bullet points or sub-headings so that it is easier to derive key ideas from later on.

It was felt that the exchange of field notes was a useful exercise to gain a different perspective on the activity but important pieces of information were sometimes left out. Conversely, a fresh pair of eyes reading through the field notes occasionally pulled out new pieces of data that the original author might not have been able to spot.

When extracting the data onto the individual post-it notes, there was often difficulty with the level of detail that was recorded onto each note. For example, writing a single word that could have multiple meanings depending on how it is interpreted. There needs to be a pre-agreed level of detail for writing each note so that they can be easily shared and discussed.

4.6.3 Physical Access to the Affinity Boards. During the workshop, there was a contrast between the earlier tasks where the participants were working in small groups vertically on a wall and the later stages where the large group was working horizontally on a tabletop. The horizontal layout created a physical barrier of people so some had an advantage over others by it being easier to read the notes based on the position that they were in. This made some people quite dominant so they were blocking the view of others who were trying to read the notes. A solution to this problem would be to break down the large group across multiple tables so there is equal access and people are not continually jostling for the best position.

4.6.4 Information Overload. One of the interviewees stated that they had felt almost panicked at the huge amount of information that was laid out on the tabletop and it took real effort to re-engage with meaning behind each note. As there was so much to take in at once, the meaning of all the words had quickly started to disappear. A way to resolve this might be to start turning some of the notes into visual representations. This could help to make some of the concepts more approachable and free up thought process to concentrate on the ideas being represented instead of trying to always understand the meaning of the words.

During the final stages, there was often a lot of duplication in the data. It would be worthwhile to start stripping out some of the extraneous the notes and only work with the most important ones instead. This would help the key ideas come to the surface so they could be concentrated on and not get distracted by things of less importance.

4.6.5 Working Across Different Axis. At the end of the workshop, when the group had zoomed out of the final exercise, a lot of the information had been internalized at this point and lead to constructing a physical model with multiple dimensions of meaning. This started off with 2 dimensions but then it was realized that 3 dimensions would be necessary. The participants thought that it would be too difficult to think in 3 dimensions but they could quite easily work across 2 planes at a time so the analysis could be broken down and then rebuilt again afterwards. For example, with the person 
to people axis and tech to non-tech, these could be broken down into four separate quadrants and then the teams could work on these individually so they are not trying to think about too many things at once.

\section{Field Studies 2 and 3}

The method has been applied an additional two times both in education settings. These were at the University of Arts Zurich, Switzerland and again at the University of Dundee, Scotland with an alternative class. We will briefly describe the different conditions in comparison to the first field study and discuss how the methodology was refined based on our previous understanding.

\subsection{Field Study 2}

In Zurich, the same method was followed although the group who took part had less experience of using fieldwork techniques.

5.1.1 Participants. Eleven students on a Masters of Arts in Design participated in the study ( 8 male, 3 female). The participants were grouped into 4 separate teams with a theme assigned to each team.

5.1.2 Location. The town centre of Zurich, Switzerland was used as the location to carry out the fieldwork activity. The transactions team was located at the budget shopping centre ShopVille - RailCity; the interactions team was located at the upmarket shopping centre Globus Bellevue; the conversations team was based at the Masoala Rainforest Hall and the connections team was based at the Kunsthaus Zürich Museum for Modern Art. We did not have to seek permission to make observations in any of these locations and we were able to take photographs if required.

5.1.3 Materials. Each group was provided with a briefing card alone instead of the additional note and sketchbooks that were previously supplied. Participants were given additional hints on the briefing cards instructing them to assign roles for each team member between note taking, sketching and taking photographs. The cards also stated that the questions set out were only suggested and asked the participants to note down any additional questions that emerged as their observations progressed. A checklist was provided to the facilitator of the workshop to ensure that all the correct steps were followed for each phase of the methodology.

5.1.4 Fieldwork. The fieldwork phase of activity took place over two days, which allowed the teams to make a first attempt at observing and then return back to the studio to discuss what they had seen. This gave the group an opportunity to reflect on their progress early on and decide how to refine their fieldwork strategy further.

5.1.5 Results. Each of the teams produced a presentation of their findings following the final workshop phase of activity. This was a very useful additional task as it forced the group to consolidate the material they had gathered into a series of insights. 
The conversations team even went as far as creating a short video featuring footage they had shot whilst out in the field. This video helped to convey their findings in an informative and accessible way.

\subsection{Field Study 3}

The method was applied an additional time with a different set of Design Ethnography students.

5.2.1 Participants. Fourteen students on the Masters of Design Ethnography participated in the study ( 9 male, 5 female). The participants were grouped into 4 separate teams and a theme was assigned to each team.

5.2.2 Location. The city centre of Edinburgh, Scotland was selected as the location to make the fieldwork observations. The teams were not assigned to any specific locations so they were given the freedom to select where would be most suitable for their particular theme. The only constraints for the participants were they could not go into any interior public spaces (railway station or shopping centre for example) as no prior permission had been sought.

5.2.3 Themes. The themes were altered slightly so the connections theme was replaced with a consumers theme and conversations was replaced with environments. A workshop prior to the fieldwork activity was also held that asked the teams to define their own questions based around each theme before going out into the field. Although this gave the teams greater ownership of the research questions, it made the task significantly more difficult.

5.2.4 Materials. As the teams had to define their own individual locations and themes prior to going out into the field, they were not provided with a briefing card as in the previous studies. However, each team was given a pocket-sized video camera that could be used to help document their observations.

5.2.5 Results. Following the fieldwork, the group was asked to create a short video to communicate their findings based on the observations they had analysed. It was useful to have this final piece of work to help summarise their analysis of all of the gathered material. It was also interesting to contrast how the group progressed without any tangible support materials when going out into the field. They seemed to be too ambitious with how much they were hoping to observe in the space of one day. However, the learning experience was improved greatly as without the support materials, the participants had to consider a lot more about how they planned their observations prior to the fieldwork activity.

\section{Conclusion and Future Work}

The method we have proposed sets out a new way of using multiple novice fieldworkers and so gaining multiple viewpoints when engaging with a design 
problem. We have been able to initially break down our problem into smaller parts and divide these up amongst a large group of novice fieldworkers. Such a group can explore the field setting and gradually co-create a rich understanding that cannot usually be gained by lone fieldworker observing in such a short time frame.

There is a clear need for tools to help collect, manage and analyse the fieldwork data when there is such a large group of people involved in the process. We have used an affinity mapping method with physical post-it noting sessions to do this but this has a number of limitations in relation to providing context around each of the pieces of data. Some existing tools, such as Prezi (prezi.com), offer some of the functionality required but lack the ability to manage multiple sources of material and deal with a variety of configurations of the gathered data.

There has been work investigating the use of tablet PCs [14] as a replacement for the existing lo-fidelity material in affinity diagramming. Another potential solution could be to use a multi-touch, multi-user technology platform that enables many people to work around the problem at the same time. This would help to alleviate some of the issues that we have identified and also enable sessions to be both colocated and take place between different locations.

Of course it should be noted that this method would not suit all types of problem. For instance, our approach to field data gathering would not be suitable for going into a small space such as a home with 14 people all observing the same thing. This would be uncomfortable for both the fieldworkers and subject! It would also completely disrupt the normal activity going on in such an intimate setting. Working across different types of public spaces provides enough room for a large number of fieldworkers to make their observations without continually overlapping with each other. Also, it is easier for the fieldworkers to make their observations without having too much effect on the normal behaviour of people within such a large space.

As our methodology has been greatly refined using the three field studies we have discussed in this paper, the next logical step is to apply it in anger within an industry context. It is here that the method could be of real benefit as the issues related to limited time and resources for carrying out lengthy field studies are much more pronounced. This is also the setting where any insights the methodology generates can be robustly evaluated to see how much value they can bring to the organization through gaining an understanding of their end users.

Acknowledgments. This research project is jointly funded by industry sponsor NCR and the Northern Research Partnership. We are grateful to the MSc Design Ethnography students who participated in the study. We thank the reviewers for their constructive comments.

\section{References}

1. Benkler, Y.: Coase's Penguin, or, Linux and the Nature of the Firm. The Yale Law Journal 112(3), 369-446 (2002)

2. Weber, S.: The success of open source. Harvard University Press, Cambridge (2004)

3. Howe, J.: The Rise of Crowdsourcing. Wired Magazine (2006) (June 14)

4. Salvador, T., Mateas, M.: CHI 1997 tutorial: Design Ethnography: Using Custom Ethnographic Techniques to Develop New Product Concepts (1997) 
5. Millen, D.R.: Rapid ethnography: time deepening strategies for HCI field research. In: DIS 2000: Proceedings of the 3rd Conference on Designing Interactive Systems, pp. 280-286. ACM, New York (2000)

6. Myers, M.: Investigating information systems with ethnographic research. Communication of the AIS 2, 1-20 (1999)

7. Zeisel, J.: Inquiry by design: environment behavior neuroscience in architecture, interiors, landscape, and planning, Rev. ed. W.W. Norton \& Company, New York (1979)

8. IDEO (Firm), IDEO method cards. IDEO, Palo Alto (2003)

9. Strauss, A.: Basics of qualitative research: techniques and procedures for developing grounded theory, 2nd edn. Sage Publications, Thousand Oaks (1998)

10. Kawakita, J.: KJ method: A scientific approach to problem solving. Kawakita Research Institute, Tokyo (1975)

11. Beyer, H.: Contextual design: defining customer-centered systems. Morgan Kaufmann, San Francisco (1998)

12. Pang, A.S.: Thinking Big: Large Media, Creativity, and Collaboration. Parsons Journal For Information Mapping III(1), 1-12 (2011)

13. Gault, P., et al.: Urban Traces - A Pilot Study into Public Space (2010) (unpublished document)

14. Mohamedally, D., Zaphiris, P.: Categorization Constructionist Assessment with SoftwareBased Affinity Diagramming. 25(1), 22-48 (2009) 